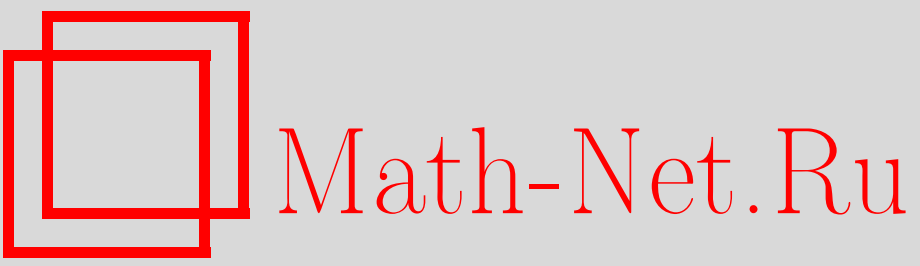

Ю. И. Поляков, Теория возмущений для частицы в одномерном, центрально- и аксиально-симметричном полях, ТМФ, 1997, том 112, номер 1, 119-123

DOI: https://doi.org/10.4213/tmf1032

Использование Общероссийского математического портала Math-Net.Ru подразумевает, что вы прочитали и согласны с пользовательским соглашением

http://www . mathnet.ru/rus/agreement

Параметры загрузки:

IP: 35.173 .219 .12

26 апреля 2023 г., 15:49:51 
ТЕОРЕТИЧЕСКАЯ

И МАТЕМАТИЧЕСКАЯ

ФИЗИКА

Том 112, № 1

июль, 1997

Ю.И. Поляков *

\section{ТЕОРИЯ ВОЗМУЩЕНИЙ ДЛЯ ЧАСТИЦЫ \\ В ОДНОМЕРНОМ, ЦЕНТРАЛЬНО- И АКСИАЛЬНО-СИММЕТРИЧНОМ ПОЛЯХ}

Получена теория возмущений, в которой все поправки к невозмущенному состоянию определяются волновой функцией этого состояния. Сформулировано новое условие применимости теории возмущений. На простом примере рассмотрена связь с теорией возмущений Рэлея-Шредингера, откуда, в частности, следует аналитическое выражение для фигурирующего в ней ряда.

В теории возмушений Рэлея-Шредингера для вычисления поправок к состоянию $|n\rangle$ с энергией $E_{n}$ необходимо знать волновые функции и энергии всех остальных невозмушенных состояний. В рассматриваемых в дальнейшем случаях одномерного, центрально-симметричного или аксиально-симметричного полей уравнение Шредингера сводится к уравнению вида

$$
\widehat{\mathcal{L}} y=y^{\prime \prime}+\varphi(x) y=0
$$

а поправки по теории возмушений определяются уравнением

$$
\widehat{\mathcal{L}} y=f(x, \alpha)
$$

При этом в $f$ входят в качестве параметров $\alpha$ соответствуюшие поправки для энергии. Как известно, все решения неоднородного уравнения $\left(1^{\prime}\right)$ определяются всего одним решением однородного уравнения (1), поэтому и все поправки должны выражаться только через волновую функцию невозмушенного состояния. Эта идея развита в работе [1] для центрально-симметричного поля. Мы используем другой подход, включаюший также случай одномерного и аксиально-симметричного полей. Невозмушенная функция удовлетворяет граничным условиям

$$
y_{0}(a)=y_{0}(\infty)=0
$$

где $a=-\infty$ для одномерного поля, а в двух других случаях $a=0$.

${ }^{*}$ Софийский университет Святого Климента Охридского, София, Болгария 
1. Одно специальное решение ${ }^{*} y$, удовлетворяющее начальным условиям $y(\xi)=$ $y^{\prime}(\xi)=0$, дает принцип Дюамеля:

$$
{ }^{*} y(x, \xi)=\int_{\xi}^{x} u\left(x, x^{\prime}\right) f\left(x^{\prime}, \alpha\right) d x^{\prime}, \quad u\left(x, x^{\prime}\right)=y_{02}(x) y_{01}\left(x^{\prime}\right)-y_{01}(x) y_{02}\left(x^{\prime}\right) .
$$

Здесь $y_{01}=y_{0}$ и $y_{02}-$ два линейно независимых решения (1) с единичным вронскианом, откуда следует, что

$$
y_{02}(x)=y_{0}(x) \int_{\bar{x}}^{x} \frac{d x^{\prime \prime}}{y_{0}^{2}\left(x^{\prime \prime}\right)} \quad \forall x .
$$

Учитьвая (3), можно переписать решение (2) в виде

$$
{ }^{*} y(x, \xi)=y_{0}(x) \int_{\xi}^{x} \frac{d x^{\prime \prime}}{y_{0}^{2}\left(x^{\prime \prime}\right)} \int_{\xi}^{x^{\prime \prime}} y_{0}\left(x^{\prime}\right) f\left(x^{\prime}, \alpha\right) d x^{\prime}
$$

Общее решение уравнения $\left(1^{\prime}\right)$ с учетом (2) записывается следуюшим образом:

$$
y(x, p, q)=-y_{01}(x) \int_{p}^{x} d x^{\prime} y_{02}\left(x^{\prime}\right) f\left(x^{\prime}, \alpha\right)+y_{02}(x) \int_{q}^{x} d x^{\prime} y_{01}\left(x^{\prime}\right) f\left(x^{\prime}, \alpha\right),
$$

где $p$ и $q$ - произвольные постоянные. При этом ${ }^{*} y(x, \xi)=y(x, \xi, \xi)$.

2. Рассмотрим теперь граничную задачу

$$
\widehat{\mathcal{L}} y=f(x, \alpha), \quad y(a)=y(\infty)=0
$$

и предположим, что $y_{0}$ удовлетворяет обоим граничным условиям, а $y_{02}-$ ни одному из них. Это предположение определяет постоянную $q$, которая равна $a$, и параметр $\alpha$ в $\left(3^{\prime}\right)$ :

$$
\int_{a}^{\infty} d x^{\prime} y_{0}\left(x^{\prime}\right) f\left(x^{\prime}, \alpha\right)=0
$$

Решение задачи (4) имеет вид

$$
y(x, p, a)=-y_{01}(x) \int_{p}^{x} d x^{\prime} y_{02}\left(x^{\prime}\right) f\left(x^{\prime}, \alpha\right)-y_{02}(x) \int_{x}^{\infty} d x^{\prime} y_{01}\left(x^{\prime}\right) f\left(x^{\prime}, \alpha\right) .
$$

3. Уравнение Шредингера в рассматриваемых полях сводится к одномерному. Пусть поле имеет вид $V=V_{0}+\varepsilon v$, где $\varepsilon v$ - малое возмущение. Для фиксированного состояния, индекс которого $n$ для краткости опускаем, $E=E^{0}+\varepsilon E^{(1)}+\varepsilon^{2} E^{(2)}+\cdots$, $y=y_{0}+\varepsilon y^{(1)}+\varepsilon^{2} y^{(2)}+\cdots$, где $E-$ энергия, а $y, x^{1 / 2} y$ и $x^{-1} y-$ волновые функции одномерного и соответственно радиальных уравнений (в последних двух случаях $x$ - радиальная координата). Уравнения для $E^{(k)}$ и $y^{(k)}$ имеют вид $\left(1^{\prime}\right)$ с $\varphi=\frac{2 m}{\hbar^{2}}\left(E^{0}-V_{0}\right)-U_{c}(x)$ $\left(U_{c}\right.$ - центробежный потенциал [2]) и $f=f_{k}$ :

$$
\begin{aligned}
f_{1} & =2 \Delta v y_{0}, \quad f_{2}=2 \Delta v y^{(1)}-2 E^{(2)} y_{0}, \quad \ldots, \\
\Delta v & =v(x)-E^{(1)}=v(x)-\left\langle y_{0}|v| y_{0}\right\rangle .
\end{aligned}
$$


Функция $y_{0}$ нормирована, а на $y$ наложено условие $\left\langle y_{0} \mid y\right\rangle=1$, откуда следует, что

$$
\begin{aligned}
\left\langle y_{0} \mid y^{(k)}\right\rangle & =\int_{a}^{\infty} d x y_{0}(x) y^{(k)}(x)=0, \quad k>0, \\
E^{(k)} & =\left\langle y_{0}|v| y^{(k-1)}\right\rangle .
\end{aligned}
$$

Теория возмушений Рэлея-Шредингера основана на разложении $y^{(k)}$ по всем функциям $y_{0 n^{\prime}}$ невозмушенных состояний $n^{\prime}$. В нашем случае решения для $y^{(k)}$ и $E^{(k)}$ даются формулами (6)-(9) и выражаются только через $y_{0}$. Согласно (6)

$$
y^{(1)}(x)=-2\left[y_{0}(x) \int_{p}^{x} d x^{\prime} y_{0}\left(x^{\prime}\right) y_{02}\left(x^{\prime}\right) \Delta v\left(x^{\prime}\right)+y_{02}(x) \int_{x}^{\infty} d x^{\prime} y_{0}^{2}\left(x^{\prime}\right) \Delta v\left(x^{\prime}\right)\right],
$$

а постоянная $р$ определяется условием (8). В центральных полях первый интеграл в (10) имеет смысл при $p=a=0$, если $\lim _{x \rightarrow 0} x^{2} v(x)=0$. Поэтому в соответствии с (10) имеем

$$
y^{(1)}(x)=c y_{0}(x)+{ }^{*} y^{(1)}(x, 0)=y_{0}(x)\left[c+F_{1}(x)\right],
$$

где

$$
F_{1}(x)=\int_{0}^{x} \frac{F\left(x^{\prime}\right) d x^{\prime}}{y_{0}^{2}\left(x^{\prime}\right)}, \quad F(x)=2 \int_{0}^{x} y_{0}^{2}\left(x^{\prime}\right) \Delta v\left(x^{\prime}\right) d x^{\prime} .
$$

Из (5) следует, что $F(\infty)=0$. Постоянная $c$ определяется условием $(8)$ при $k=1$, так что

$$
y^{(1)}(x)=y_{0}(x) \Delta F_{1}(x), \quad \Delta F_{1}(x)=F_{1}(x)-\bar{F}_{1}, \quad \bar{F}_{1}=\int_{0}^{\infty} y_{0}^{2}(x) F_{1}(x) d x .
$$

Согласно (9) для $k=2$ имеем

$$
\begin{aligned}
E^{(2)} & =\left\langle y_{0}|v| y^{(1)}\right\rangle=\int_{0}^{\infty} y_{0}^{2}(x) v(x)\left(F_{1}(x)-\bar{F}_{1}\right) d x=\int_{0}^{\infty} y_{0}^{2}(x) \Delta v(x) F_{1}(x) d x= \\
& =\frac{1}{2} \int_{0}^{\infty} F^{\prime}(x) F_{1}(x) d x=-\frac{1}{2} \int_{0}^{\infty} \frac{F^{2}(x) d x}{y_{0}^{2}(x)}<0 .
\end{aligned}
$$

4. Рассмотрим одномерный случай: $a=-\infty$. Обычно произведение $y_{0} y_{02}$ отлично от 0 при $x \rightarrow-\infty[3]$, так что (10) не имеют смысла при $p=-\infty$. Поэтому положим $p=x_{0}$, где $x_{0}-$ произвольная точка в области локализации $y_{0}$, и запишем $(10)$ в виде

$$
\begin{gathered}
y^{(1)}(x)=y_{0}(x)\left[c-2 \int_{x_{0}}^{x} d x^{\prime} y_{0}\left(x^{\prime}\right) y_{02}\left(x^{\prime}\right) \Delta v\left(x^{\prime}\right)\right]+y_{02}(x) F(x) \\
F(x)=2 \int_{-\infty}^{x} d x^{\prime} y_{0}^{2}\left(x^{\prime}\right) \Delta v\left(x^{\prime}\right)
\end{gathered}
$$

Преобразуем первый интеграл, полагая $y_{02}=z y_{0}$, где $z$ определяется с помощью $\left(3^{\prime}\right)$ :

$$
\begin{aligned}
-2 y_{0} \int_{x_{0}}^{x} d x^{\prime} z\left(x^{\prime}\right) y_{0}^{2}\left(x^{\prime}\right) \Delta v\left(x^{\prime}\right) & =-y_{0} \int_{x_{0}}^{x} z\left(x^{\prime}\right) d F= \\
& =-y_{02} F+y_{0}(F z)_{x=x_{0}}+y_{0} \int_{x_{0}}^{x} \frac{F\left(x^{\prime}\right) d x^{\prime}}{y_{0}^{2}\left(x^{\prime}\right)} .
\end{aligned}
$$


Включая $(z F)_{x=x_{0}}$ в $c$, получим

$$
y^{(1)}(x)=y_{0}(x)\left[c+F_{1}(x)\right], \quad F_{1}(x)=\int_{x_{0}}^{x} \frac{F\left(x^{\prime}\right) d x^{\prime}}{y_{0}^{2}\left(x^{\prime}\right)},
$$

или, определяя $c$ из (13) при $k=1$,

$$
\begin{gathered}
y^{(1)}(x)=y_{0}(x) \Delta F_{1}(x), \quad \Delta F_{1}(x)=F_{1}(x)-\bar{F}_{1}, \\
\bar{F}_{1}=\left\langle y_{0}\left|F_{1}\right| y_{0}\right\rangle=\int_{-\infty}^{\infty} y_{0}^{2}(x) F_{1}(x) d x .
\end{gathered}
$$

Повторяя вывод формулы (13), находим

$$
E^{(2)}=-\int_{-\infty}^{\infty} \frac{F^{2}(x) d x}{y_{0}^{2}(x)}
$$

Эта формула, как и $\left(14^{\prime}\right)$, не зависит от выбора $x_{0}$. Аналогично получаются все поправки. Они выражаются только через $y_{0}$.

Формулы типа (12)-(15) особенно важны, когда набор $y_{n}$ не образует полной системы и традиционная теория возмушений неприменима. Однако и в противном случае сравнение этих формул с соответствуюшими рядами дает аналитическое представление для последних (см. ниже (18)).

5. Получим условие применимости теории возмушений. Пусть $L$ - характерный размер области локализации $y_{0}$ в единицах, в которых $m=\hbar=\bar{V}_{0}=1\left(\bar{V}_{0}-\right.$ характерная величина $\left.V_{0}\right)$. Из условия нормировки следует, что $y_{0} \sim L^{-1 / 2}$, а из единичности вронскиана имеем $y_{02} \sim L^{3 / 2}$ при $x \sim L$. При $x \sim L$ должно быть $\varepsilon y^{(1)} \ll y_{0}$ и, оценивая первый интеграл в $(10)$, находим $L^{2} \varepsilon \bar{v} \ll 1$ или в обычных единицах

$$
\frac{m L^{2} \varepsilon \bar{v}}{\hbar^{2}} \ll 1
$$

Это условие имеет простой физический смысл: $\varepsilon v$ должно быть мало по сравнению с неопределенностью потенциальной энергии $V_{0}$, вызванной локализацией частицы в области $x \sim L$.

6. В заключение в качестве примера рассмотрим трехмерный изотропный осциллятор и выгислим возмушение основного состояния с $l=0$ при $v=x^{3}$. Радиальные функции $y_{0 n}=\sqrt{2} \psi_{2 n+1}$, где $\psi_{k}$ - волновые функции одномерного осциллятора $\left(\psi_{2 n}\right.$ не удовлетворяют граничному условию при $x=0$; введение множителя $\sqrt{2}$ связано с тем, что отбрасывается полуось $x<0$ ). В рассматриваемом случае

$$
y_{0}=c x \exp \left(-x^{2} / 2\right), \quad c^{2}=4 / \sqrt{\pi} \quad(m=\hbar=\omega=1) .
$$

Найдем $y^{(1)}$ по формуле (12) и по теории Рэлея-Шредингера.

Так как

$$
\left\langle y_{0}\left|x^{3}\right| y_{n}\right\rangle=2 \int_{0}^{\infty} x^{3} \psi_{1}(x) \psi_{2 n+1}(x) d x=\frac{4}{\sqrt{\pi}}(-1)^{n} \frac{(2 n+1) !}{2^{n} n !^{2}} \neq 0
$$


(из-за нечетности возмушения), то по теории Рэлея-Шредингера получаем

$$
y^{(1)}=-\frac{2}{\sqrt{\pi}} e^{-x^{2} / 2} \sum_{n=1}^{\infty} \frac{(-1)^{n}(2 n+1) !}{2^{n} n !^{2} n} H_{2 n+1}(x),
$$

где $H_{k}(x)$ - полиномы Эрмита.

При использовании формулы (12) имеем: $E^{(1)}=c^{2}$,

$$
\begin{aligned}
F & =2 c^{2} \int_{0}^{x} d t t^{2} e^{-t^{2}}\left(t^{3}-c^{2}\right)=2 c^{2}\left(J_{5}-c^{2} J_{2}\right), \\
F_{1} & =2 \int_{0}^{x} \frac{d t}{t^{2}} e^{t^{2}}\left(J_{5}(t)-c^{2} J_{2}(t)\right)
\end{aligned}
$$

где

так что

$$
J_{n}(x)=\int_{0}^{x} t^{n} e^{-t^{2}} d t=\frac{n-1}{2} J_{n-2}(x)-\frac{1}{2} x^{n-1} e^{-x^{2}},
$$

$$
\begin{gathered}
J_{0}(x)=\frac{\sqrt{\pi}}{2} \operatorname{erf}(x), \quad J_{1}(x)=\frac{1}{2}\left(1-e^{-x^{2}}\right), \\
J_{2}(x)=\frac{1}{2}\left(J_{0}-x e^{-x^{2}}\right), \quad J_{5}(x)=2 J_{1}-x^{2}\left(1+\frac{1}{2} x^{2}\right) e^{-x^{2}} .
\end{gathered}
$$

Соответственно формула (17) после интегрирования по частям дает

$$
F_{1}(x)=4 \int_{0}^{x} e^{t^{2}} \operatorname{erfc}(t) d t+\frac{2}{x}\left(1-\frac{2 x}{\sqrt{\pi}}-e^{x^{2}} \operatorname{erfc}(x)\right)-2 x-\frac{x^{3}}{3}
$$

откуда

$$
y^{(1)}=\frac{2 x}{\pi^{1 / 4}} e^{-x^{2} / 2}\left[F_{1}(x)-\bar{F}_{1}\right], \quad \bar{F}_{1}=\frac{4}{\sqrt{\pi}} \int_{0}^{\infty} x^{2} e^{-x^{2}} F_{1}(x) d x .
$$

Сравнение с (16) дает аналитическое выражение для записанного там ряда.

\section{Список литературы}

[1] В. С. Поликанов. ЖЭТФ. 1967. Т. 52. № 5. С. 1326.

[2] А. Мессиа. Квантовая механика. Гл.XVI. М.: Наука, 1979.

[3] М. В. Федорюк. Обыкновенные дифференциальные уравнения. Гл.7. М.: Наука, 1985.

Поступила в редакцию 25.III.1996 г., после доработки 10.I.1997 г.

\section{Yu. I. Polyakov \\ PERTURBATION THEORY FOR PARTICLE \\ IN ONE-DIMENSIONAL, CENTRAL- OR AXIAL-SYMMETRIC FIELDS}

Perturbation theory for which all corrections to a nonperturbed state are defined by its wave function is obtained. A new applicability condition for this theory is formulated. By considering a simple example comparison with the Rayleigh-Schrödinger perturbation theory giving an analytical expression for the corresponding series is performed. 